\title{
Indexing Coded Stripe Patterns Based on De Bruijn in Color Structured Light System
}

\author{
Cheng Han \\ College of Computer Science and Technology, \\ Changchun University of Science and Technology \\ Changchun, Jilin, China, 0431-85583332 \\ hancheng@cust.edu.cn
}

\begin{abstract}
The problem of relating projected and captured stripes, here called the Indexing Problem, has proved to be difficult to overcome reliably for stripe patterns in color structured light, due to color fidelity of the projector and the camera, the ambient light, texture of measured object and other factors. In order to solve the Indexing Problem, a new method is proposed. The method extract the center color stripe based on cluster analysis at first. We convert color coded image based on De Bruijn into weighted directed digraph, then match stripe according to coded values. The proposed method is the center of colored stripes as the basic decoding units. Results are significantly more rapid, accurate and reliable than previous attempts.
\end{abstract}

Keywords-color structured light; De Bruijn coding; clustering analysis; weighted directed graph

\section{INTRODUCTION}

The reconstruction of 3D models based on structured light is a longstanding and challenging technology in computer vision, with applications in game, movie, design, and manufacturing, and so on. The controlled illumination is used to actively generate geometric correspondence between projector views and camera views as basic step for 3D reconstruction. Now, various structured light systems for 3D reconstruction have been developed with quite different characteristics $^{[1-4]}$. In some applications it is imperative that sufficient information for dense reconstruction is acquired within the timebase of a single video frame. The coding and decoding technology directly influences speed and accuracy of reconstruction. Therefore, researchers have focused on speeding up the acquisition process by designing techniques that require only a small number of input images, in some cases even a single image. Because it can achieve real-time object reconstruction, so color structured light method is concerned by more and more people. But the problem of relating projected and captured stripes, in [5] called the Indexing Problem, has proved to be difficult to overcome reliably for stripe patterns in color structured light, due to color fidelity of the projector and the camera, the ambient light, texture of measured object and other factors ${ }^{[6]}$.

Today, various methods have been researched to solve indexing problem: in [7] the authors present an adaptive color classification method to achieve high-quality 3D reconstructions with a single-shot structured light system

Key Projects in the National Science \& Technology Pillar Program of China (2009BAE69B00)

\author{
Zhengang Jiang \\ College of Computer Science and Technology, \\ Changchun University of Science and Technology \\ Changchun, Jilin, China, 0431-85583332 \\ jiangzhengang@cust.edu.cn
}

without the need of dark laboratory environments; in [8] the authors present a framework to capture 3D models of faces in high resolutions with low computational load using only two pictures of the face, one illuminated with a color stripe pattern and one with regular white light; in [9] a novel, multi-pass dynamic programming algorithm is proposed to eliminate global smoothness assumptions and strict ordering constraints present in previous formulations; in [5] the authors use the maximum spanning tree of a graph defining potential connectivity and adjacency in captured stripes to solve the indexing problem for uncoded stripe patterns.

Because De Bruijn sequence has determinability and reproducibility, so color structured light coding method using De Bruijn pseudo-random sequence has become one of the most widely used methods in $3 \mathrm{D}$ reconstruction system ${ }^{[10]}$. But color variety is more in stripe pattern, color interference between neighboring feature points is serious. The existing algorithms complete match of pixels on the stripes, thus it takes a long time to complete the stripe matching, and often appears errors in the bad pixels and borderline of occlusion area. In this paper a new method is presented to solve the indexing problem for color coded stripe patterns. We make the stripe as decoding unit, convert color structured light image based on De Bruijn into weighted directed digraph, then match stripe according to coded values.

\section{3D RECONSTRUCTION WITH COLOR STRIPE PATTERNS}

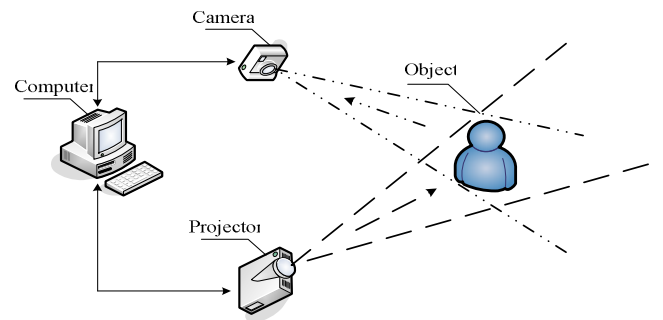

Figure 1. Structure of 3D reconstruction system based on sturctured light

In general, 3D reconstruction system based on color structured light is similar to a passive stereo vision system, one of the cameras is replaced by a projector, as shown in Figure 1. This method is mainly divided into calibration of camera and projector, coding and decoding, and calculation of the 3D information. By projecting certain type of patterns, the 
correspondence of the images can be easily identified, and depth information can be retrieved by a simple triangulation technology.

\section{A. Calibration of camera and projector}

Calibration of camera and projector is necessary step in $3 \mathrm{D}$ reconstruction based on structured light in order to extract metric information from 2D images. In the calibration process, the world coordinate system must be firstly established. After calibration is completed, it can obtain homography matrixes of camera and projector, whose coefficients are explicit functions of the camera and projector parameters. If camera and projector are properly calibrated, the world coordinates of the illuminated points can be calculated by using triangulation formulas, as has been done by homography matrixes. Calibration precision is critical to $3 \mathrm{D}$ reconstruction, because calibration error of every coefficient will result in that the reconstruction work can not achieve perfect result.

Camera calibration has been extensively studied over the years. A camera is often described by a pinhole model, with intrinsic parameters including focal length, principle point, pixel skew factor, and pixel size; and extrinsic parameters including rotation and translation from a world coordinate system to a camera coordinate system. To obtain intrinsic parameters of the camera, a standard black-and-white checkerboard is usually used, world coordinate system is establishment, as shown in Figure 2. The world-to-image perspective transformation matrix and the distorted coefficient are estimate using at least six non-coplanar world points and their corresponding image projection points ${ }^{[11]}$.

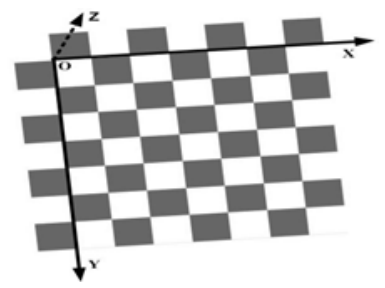

Figure 2. Calibration image and world coordinate system

A projector can be regarded as the inverse of a camera, because it projects images instead of capturing them. In this research, we propose a method that enables a projector to "capture" images like a camera, thus making the calibration of a projector essentially the same as that of a camera, which is well established.

For geometric calibration, we image a checkerboard textured plane in a variety of poses. For each pose, we also project a distinct checkerboard pattern onto the plane and take an additional image. The images taken without the projected pattern are used to estimate the camera intrinsic and plane poses. For the remaining images, we can compute the 3D coordinates of the projected pattern features and thus calibrate the projector.

\section{B. Color Stripe Patterns based on De Bruijn}

The technology of color structured light coding is foundation and key in structured light $3 \mathrm{D}$ reconstruction. Good coding technology will improve precision and accuracy of image decoding. De Bruijn sequences were widely used to color stripe patterns and multi-slit patterns by mapping every symbol of the alphabet with a certain color. The most powerful and developed technique in this field was presented by Zhang et. $\mathrm{al}^{[9]}$. A color structured light coded image of De Bruijn sequence of 7 elements 3 grades was generated using random and certainty ${ }^{[12]}$, as shows in Figure 3 . The normalized RGB values of seven colors are $(0,0,1),(0,1,0),(0,1,1),(1,0,0)$, $(1,0,1),(1,1,0),(1,1,1)$. The color background of image is black, width of each color stripe is a pixel; the interval of two adjacent color stripes is 3 pixels. The De Bruijn sequence used was generated with the constraint that two consecutive stripes could not have the same color. The main drawback of this technique is the large number of required colors.

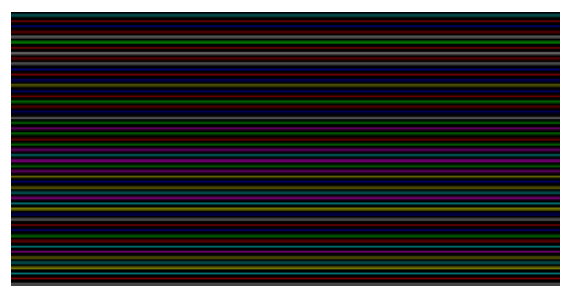

Figure 3. Color coded stripe patterns based on De Bruijn

\section{World Coordinates Calculation}

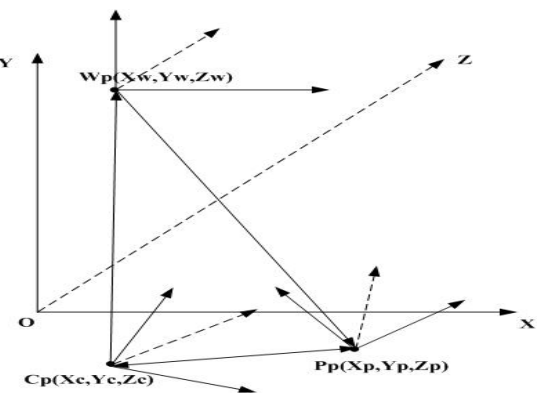

Figure 4. Solution model of three-dimensional information

In order to reconstruct the high-precision model, the world coordinates of every feature points are evaluated according to two-dimensional information in image, homography matrixes of camera and projector, and optical triangulation. Optical triangulation has been an active area of research for decades, but there are calculation errors of the angle of the camera and projector and other. So, unconstrained method is used to improve accuracy of the feature points in article. After matching relation of every point between decoded image and coded image is ensured according to coded values, 3D information of feature points are calculated using homography matrixes and maximum likelihood method, as shown in Figure 4.

\section{INDEXING CODED STRIPE PATterns BASED ON DE BRUIJN}

The color horizontal stripes that are coded using De Bruijn sequence are projected on the measured object through the projector to generate the modulated projection image. If the 
surface of object is flat, then the captured stripe is perfectly horizontal and appears in every image column. If the surface of object is sufficiently smooth, a stripe will across a number of columns in the captured image. The indices can be absent from certain columns due to occlusions or weak surface reflection. In order to solve Indexing Problem, a new decoding based weighted directed graph is proposed.

\section{A. Extraction of color stripes}

Due to the image was modulated by measured object, and resolution and position of camera and projector, the width of the stripe in shot color structured light image through camera can be changed, therefore it need to extract each center color stripe, i.e. extracting center color stripe.

In order to accurately extract the center color stripe, at first the color coded image was grayed and normalized. After filtering, based on form of waveform every column elements of image matrix, found location each peaks and troughs in waveform, then according to two adjacent peaks and troughs of the calculated middle between peaks and troughs position. Finally, in the matrix according to each two adjacent position to find the first maximum or minimum gray value, the pixel is a feature point of coded image, all feature points constitute the central stripe.

As the projector and camera configuration, interference between adjacent color stripes and other reasons, reflection and absorption characteristics of object to different color stripes are different, so the ordinary color classification can not get accurate results. Therefore, we use a color classification method based on cluster analysis to determine the color of stripes ${ }^{[12]}$. The method can be applied to avoid color interference effectively in the coded structured light images containing parallel pixel lines with different colors.

\section{B. Indexing by weighted directed digraph}

We proceed to define a set of neighbours for every stripe pixel, by heading west (w), east (e), north (n) and south (s). The w-neighbour of stripe pixel $(i, j)$ is the stripe pixel in the preceding column $(j-1)$ that shares at least one corner with $(i, j)$, if such a same color pixel exists. The e-neighbour of $(i, j)$ is the stripe pixel in the next column $(j+1)$ that shares at least one corner with $(\mathrm{i}, \mathrm{j})$, if such a same color pixel exists. The nneighbour of $(i, j)$ is the very next color stripe pixel encountered when moving upwards in column $\mathrm{j}$, and the s-neighbour is the very next color stripe pixel encountered when moving downwards.

We define a we-connected group to be a sequence of $\mathrm{m}$ stripes pixels $P_{1}, \ldots, P_{m}$ such that $P_{1}$ has no w-neighbour, $P_{j}$ is the e-neighbour of $P_{j-1}$ for every $j \in\{2,3, \ldots, m\}, P_{m}$ has no eneighbour. We say that an ns-connection exists from a weconnected group a to a we-connected group $b$ if at least one pixel in $b$ is the $n$-neighbour of a pixel in a. Such a connection is denoted by the ordered pair $(a, b)$ and indicates a possible increase of 1 in the index from group a to group $b$. Due to breaks in the captured stripes some of these connections should in fact not be allowed to influence the indexing in this manner. We say $(a, b)$ is a strong ns-connection if and only if a pixel in group $b$ is the $n$-neighbour of a pixel in group a across every column that contains both $\mathrm{a}$ and $\mathrm{b}$. The condition is not met when another group of pixels lies "between" $a$ and b.

In Figure 5, for example, the w-, e-, n- and s-neighbours of pixel 6 are pixels 7, 8, 9 and 10 respectively, and n-neighbour of pixel 12 is pixel 11. The stripe pixels can be split into eleven we-connected groups, respectively as a, b, c, d, e, f, g, h, k, l, m. We say (a,b), (a,g), (b,c), (b,h), (c,d), (c,l), (d,e), (e,f), (g,h), $(\mathrm{h}, \mathrm{k}),(\mathrm{k}, \mathrm{l}),(\mathrm{l}, \mathrm{e}),(\mathrm{l}, \mathrm{m}),(\mathrm{m}, \mathrm{f})$ are all strong ns-connection, $(\mathrm{a}, \mathrm{h})$, $(\mathrm{b}, \mathrm{k}),(\mathrm{l}, \mathrm{f})$ are all ns-connection.
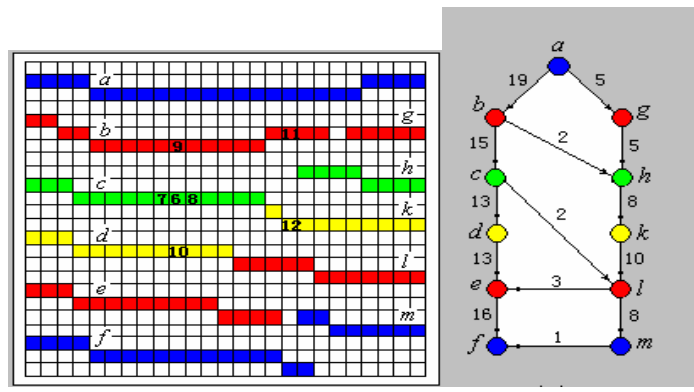

Figure 5. Illustrating color stripe pixel neighbours

The connections determine a weighted directed graph G: every we-connected group is represented as a vertex, and every strong ns-connection as a directed edge between corresponding vertices. The every edge weight is the number of columns that its corresponding ns-connection spans. If there are isolated vertexes, these vertexes are removed. Figure 6 shows the graph corresponding to the stripe pixel shown in Figure 5.

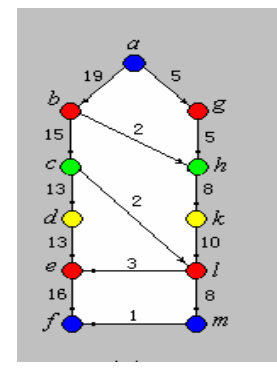

Figure 6. Strong ns-connections between we-connected groups determine a weighted directed graph.

In general $\mathrm{G}$ may not reveal a clear and unique indexing and it is possible, specifically when $G$ contains cycles, that only a subset of the edges can and should contribute. Thereby, $\mathrm{G}$ will be simplified:

If the two vertexes have at least one parent vertex, and one sub-vertex in weighted directed graph, at the same time the colors of two vertexes are same, then they are called similar vertex. The all similar vertex are simplified into one vertex. The directed edges are simplified into one edge between common parent vertex and similar vertex, its weight is sum of weights of directed edges. The directed edges are simplified into one edge between common sub-vertex and similar vertex, its weight is sum of weights of directed edges. The simplification is not completed until there are no similar vertexes in the weighted directed graph. Figure 7 shows simplified weighted directed graph. 


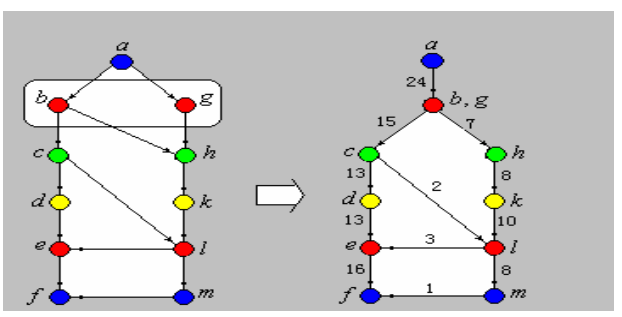

Figure 7. Simplified weighted directed graph

The color sequence of three adjacent stripes is unique in the coded image based on De Bruijn. According to color sequence of the max weights, we can accurately decode to coded image.

\section{EXPERIMENTATION AND DISCUSSION}

Color structured light coding and decoding system was composed of hardware and software. The model of used computer in hardware system is DELL DIMENSION 5150. Due to the current home projector has been very good, so ordinary home projector basically meet the system requirements for the projector. The model of used projector is EPSON EB-W6. Camera is a key component of threedimensional reconstruction system. It is used mainly to shoot coded images, the system used Canon EOS. The color coded image is projected onto measured object from projector. The camera shoots modulated encoded image, as shown in Figure 8.
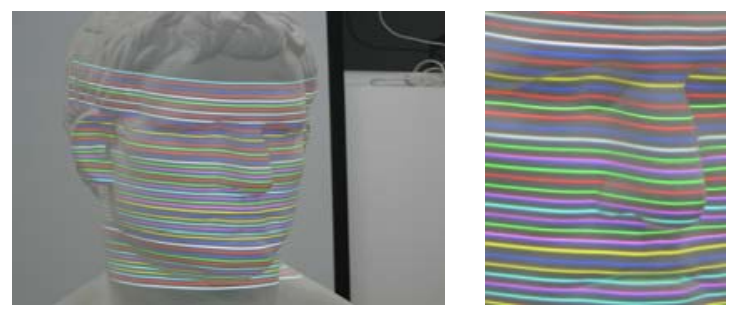

Figure 8. Plaster head model and a part of captured image

The captured image is processed using color classification based on clustering analysis. It used the four iterations to complete color classification. The color calibration error does not exceed $1.1 \%$. All of color calibration error almost appears in the nose area or the edge of the shaded area, as shown in Figure 9(a). Figure 9(b) show that the errors of color calibration and extracting stripe are eliminated by weighted directed image.

Figure 9(c)-(e) shows results of the stripe matching results, cropped for display purposes. Our indexing algorithm is applied, the lighter pixels in the figure are decoded correctly. The several experimental results show that the reliability of the decoding algorithm proposed in this paper.

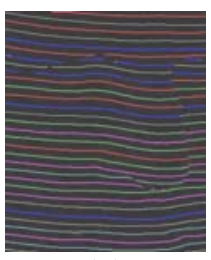

(a)

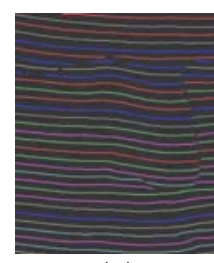

(b)

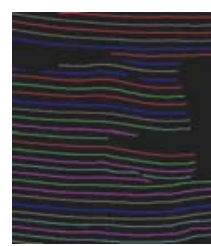

(c)

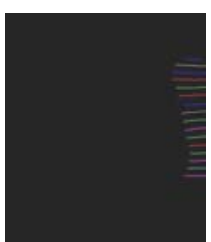

(d)

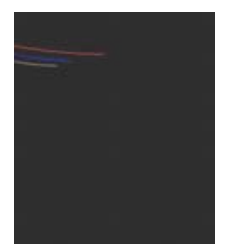

(e)
Figure 9. Decoding resulet (a) Calibration result of color (b) Calibration result base $d$ on weighted directed image (c) (f) Indexing result of code stripes

\section{ACKNOWLEDGMENT}

Key Projects in the National Science \& Technology Pillar Program of China under Grant No. 2009BAE69B00.

\section{REFERENCES}

[1] Song Zhang, Peisen S. Huang, "High-resolution, real-time threedimensional shape measurement," Optical Engineering 45(12), 123601 (December 2006)

[2] Jordi Pages, Joaquim Salvi, Christophe Collewet et al., "Optimised de bruijn patterns for one-shot shape acquisition," Image and Vision Computing, 2005, 23: 707-720.

[3] Alexandra Lathuiliere, Franck Marzani, Yvon Voisin, "Colour 3D system characterization," IEEE Industrial Electronics, IECON 2006-32 nd Annual Lonference, 2006, (6-10): 3232-3237.

[4] Weihong Su, "Projected fringe profilometry using the area-encoded algorithm for spatially isolated and dynamic object," Opt. Express, 2008, 16(4): 2590-2596.

[5] W. Brink, A. Robinson and MA Rodrigues, "Indexing Uncoded Stripe Patterns in Structured Light Systems by Maximum Spanning Trees," British Machine Vision Conference BMVC 2008, Leeds, UK, 1-4 Sep. 2008

[6] Fan Jing-tao, Han Cheng, Zhang Chao, Li Ming-xun, Bai Bao-xing, Yang Hua-min, "Study of a new decoding technology for De Bruijn structured light,”. ACTA ELECTRONICA SINICA, 2012,40(3):483488(In Chinese).

[7] Philipp Fechteler, Peter Eisert, "Adaptive Colour Classification for Structured Light Systems," Computer Vision, IET, 2009,3(2):49-59.

[8] Philipp Fechteler, Peter Eisert, Jurgen Rurainsky, "Fast and high resolution 3D face scanning," Proceedings of the 14th International Conference on Image Processing 2007[C], San Antonio, Texas, USA: IEEE, 2007.

[9] L. Zhang, B. Curless, S. M. Seitz, "Rapid shape acquisition using color structured light and multi-pass dynamic programming," Proceedings of the 1st International Symposium on 3D Data Processing, Visualization and Transmission (3DPVT), Padova, Italy, 2002: 24-26.

[10] Pagès J, Salvi J, "A new optimised De Bruijn coding strategy for structured light patterns," 17th International Conference on Pattern Recognition,2004:284-287.

[11] Fuqiang Zhou, Guangjun Zhang, "Complete calibration of a structured light stripe vision sensor through planar target of unknown orientations," Image and Vision Computring, 2005,23(1):59-67.

[12] Cheng Han, Mingxun Li, Chao Zhang and Huamin Yang, "Color Classification for Structured Light of De Bruijn Based on Clusting Analysis," Recent Advances in Computer Science and Information Engineering, Lecture Notes in Electrical Engineering, 2012,128:359:364. 